\title{
Numerical Simulation of Physical Transportation Network
}

\author{
Alexander Liang \\ The Lawrenceville School, Lawrenceville, NJ 08648, USA \\ Email: 1052231061@qq.com
}

\begin{abstract}
Prior experiments with physarum polycephalum, a plasmodial slime mold, have shown its inherent biological ability to adapt to the surroundings. When placed in an environment with food surrounding it, it builds a network of biological pipelines to the food that reinforces heavily used pipelines and withdraws those rarely used. Interestingly, the human body displays a similar ability. The development of our circulatory system is in the absence of our brain or nervous system, which suggests humans mimic low level organisms in the regard that our biological transportation systems are self-regulating and self-constructing. Like slime molds, this adaptive quality suggests that our biological networks follow a method of lowest energy consumption: energy is allocated to maintaining and constructing only the most efficient paths. Thus, the purpose of our model is to explain the biological mechanism of how organisms construct a pipeline network through a mathematical model. The model is developed using 2 main assumptions: (1) The network exists in a hexagonal grid system and (2) there only exists nodes that are sinks, sources, or neither. Borrowing Kirchhoff's Law and other electric circuit principles, we determine an energy function for the construction and maintenance of a biological pipeline network in our model dependent on the current, conductivity, and pressure within the pipelines. The method we develop in this paper is called the negative gradient flow method. Given any initial values for the pipeline, the negative gradient flow method is an algorithm for unconstrained nonlinear optimization that finds at each instant the next set of values to most minimize the function until eventually reaching the minimizer values. Accomplishing the main goal of this paper, the negative gradient flow improves upon existing models at replicating natural phenomena since it is based on the evolutionary advantage of lowest energy consumption.
\end{abstract}

Keywords: negative gradient flow, physical transportation system, energy optimization

\section{Introduction}

Predecessors have done a very interesting experiment with physarum polycephalum, a plasmodial slime mold. In this experiment, we put physarum polycephalum at the entrance of a maze and place a plate of food at the exit of a maze with multiple dead ends, leaving only one road leading to the food source. It was found that the pipeline established by this slime mold will branch at each bifurcation of the maze at the beginning. If a branch can lead to a food source, it will eventually remain. If a branch does not lead to a food source, the branch will gradually disappear. As a result, this creature solved the maze by continuously traversing it, and in the end only a single road from the entrance to the exit remained. A maze with multiple exits with food sources will lead to a network of pipelines only leading directly to those sources.

We compare the energy consumption (in unit time) in the network pipeline with the power consumption of a circuit: the flow (moisture, food, etc.) in the pipeline through the cross-sectional area per unit time is regarded as the current, the resistance in the pipeline (frictional force, shear force, etc.) is regarded as the resistance, and the difference in pressure between the two ends of the pipe is regarded as the potential difference. Conductivity is the inverse of resistance. We will record the conduction rate as $\mathrm{C}$. The energy consumption in a pipeline is $\mathrm{C}^{\gamma}$, which will be proved later on.

Experiments have proved that this biological pipeline system is an adaptive system: when there are small amounts or no amount of flow in a passage, it will gradually disappear; when there is a large amount of flow in a passage, then it will be reinforced. Likewise, when there are two competitive paths to reach the same food source, by analyzing the stability of paths, the most "fit" one can be obtained. A differential equation can be used to describe this self-adaptability: $Q$ to represent the flow rate and for a monotonically increasing function.

\section{Model establishment}

If the physarum polycephalum is placed in an environment with food surrounding it, it will "build" a network of biological pipelines to the food and obtain food through these pipelines to survive. The main purpose of our model is to explain the biological mechanism of how the slime mold constructs a biological pipeline network through a mathematical 
model, which is an important method for studying natural phenomena.

For my model, I set the environment where the physarum polycephalum is located as a regular hexagon. The slime mold is initially located at the lower left corner of the regular hexagon, which is marked as point A. The food source is randomly distributed in the regular hexagon. In order to simplify the model, the regular hexagon is "discretized": each side of the regular hexagon is divided into $\mathrm{N}$ equal parts, so that there are $\mathrm{N}+1$ nodes on each side. Then for each node, connect it to the matching nodes on the sides adjacent to the node's opposite side (both left and right), so that the entire regular hexagon is divided into a network structure by three types of parallel lines (left to right diagonal, right to left diagonal, and horizontal) as the following figure shows.

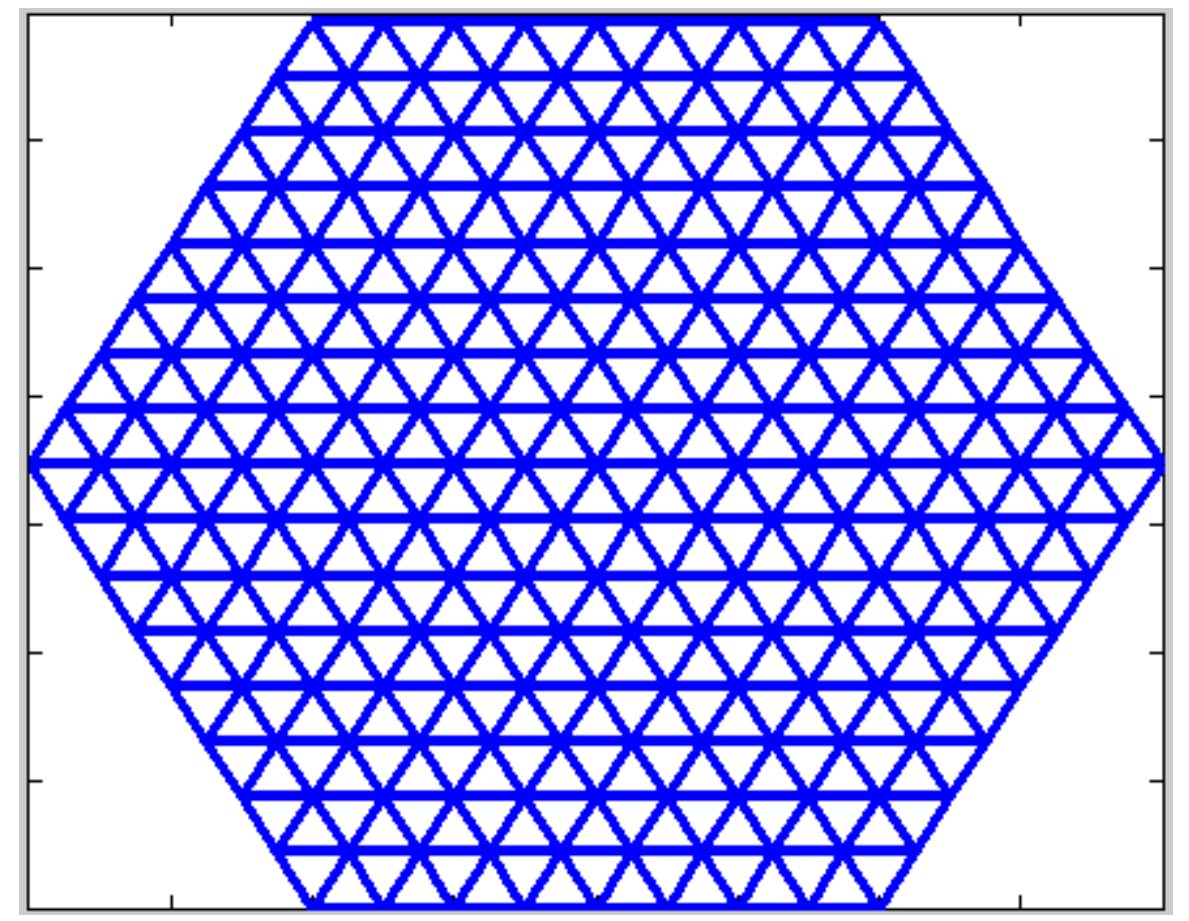

Figure 1. Sample regular hexagonal grid network

The regular hexagon has $\mathrm{N}+1$ nodes on each side and a total of $3 \mathrm{~N}^{2}+3 \mathrm{~N}+1$ nodes. Assuming that the slime mold is initially in the lower left corner, that point in the lower left corner becomes the source of the network and the biological pipeline extends outward from that point. If there is a food source on a grid point, it is called a sink because the biological pipeline extends from the source to this point.

We number the nodes of this network from bottom to top, from left to right, from 1 to $3 \mathrm{~N}^{2}+3 \mathrm{~N}+1$. As mentioned in the abstract of the paper, we compare the network to a circuit, and use $C_{i j}$ to represent the conductance between node $\mathrm{i}$ and node j. $Q_{i j}$ represents the flow between node $\mathrm{i}$ and node $\mathrm{j}$, and $\mathrm{p}_{\mathrm{i}}$ represents the pressure at node i. There is a flow between each node and its adjacent nodes. We call the algebraic value of the total outflow away from the node minus the total inflow to this node as the total flow of the node, denoted by $Q_{i}$. We stipulate that at each source, the total flow is a positive definite value; at each sink, the total flow is a negative definite value; at a node that is neither a source nor a sink, the total flow is 0 . This is consistent with the definition of sink and source, the following are the equations.

$$
Q_{k}=\sum_{i} Q_{i k}=\left\{\begin{array}{cr}
m & k \text { is the source } \\
-1 & k \text { is the sink } \\
0 & \text { else }
\end{array}\right.
$$

$\mathrm{m}$ is the number of sinks in the network. Since there is only one source in our model (node 1), the aforementioned statement ensures that the total flow in the system is $0: \sum Q_{i}=0$. The syntax $(\mathrm{i}, \mathrm{k})$ means that in our equations described above, nodes $\mathrm{i}$ and $\mathrm{k}$ are directly connected.

In the context of a circuit, we can assume that $Q_{i j}=C_{i j}\left(p_{i}-p_{j}\right)$, which also happens to be true for the model in our paper. However, we do not take this as an obvious conclusion and will derive this formula in the following text.

The problem that the model focuses on is the amount of total energy that is consumed by the operation and 
construction of the network. This energy is the sum of two components. The first energy is consumed by friction in the pipeline, which is similar to a circuit's resistance, and is called "electric power": $\sum_{(i, j)} \frac{Q_{i j}{ }^{2}}{C_{i j}}$. The sigma notation means to sum all connected nodes. This energy represents the energy consumed for the operation of the entire network. The second energy is the energy consumed to build a pipeline. The value of this energy is $C_{i j}{ }^{\gamma}$ in our context and $\gamma=0.5$, which we will prove in the following text.

We do not limit the total energy for constructing these pipelines but include it in the total energy instead to get our unconstrained model, which must be minimized.

$$
\begin{gathered}
\text { Minimize } f=\sum_{(i, j)} \frac{Q_{i j}{ }^{2}}{C_{i j}}+\lambda \sum_{(i, j)} C_{i j}{ }^{\gamma} \\
Q_{k}=\sum_{i} Q_{i k}=\left\{\begin{array}{cc}
m & k \text { is the source } \\
-1 & \text { kis the sink. } \\
0 & \text { else }
\end{array}\right.
\end{gathered}
$$

$\lambda$ is a unit of energy to construct the network of a known value. The value of $\lambda$ does not affect the structure of the terminal network.

We need to give an initial state of the slime mold biological pipeline. The most direct idea is that initially the slime mold starts from "nothing" and builds the pipeline from just one point, the source. But our subsequent research methods found that if the the initial value of $C_{i j}$ is 0 in our model, the network never forms since our unconstrained model is already minimized when $C_{i j}$ remains at 0 . No energy is consumed because no pipelines exist. Therefore, setting the initial value like this has no meaning. Secondly, the initial values for $C_{i j}$ does not affect the overall structural shape of the formed network and only affects the specific sizes of pipelines, such as the radius. For these two reasons, we take the initial value as the case where all $C_{i j}$ between connected points in Figure 2 are equal. Let them all equal 1.

\section{Determination of $\gamma$ value}

In the model established above, we mentioned that the value of $\gamma$ is 0.5 , which is derived in the following.

Assume that the pipe between any two nodes is cylindrical with length $\mathrm{L}$ and its cross section is a circle with radius $\mathrm{R}$.

Use $f$ to denote the shear force at the tube wall. The flow of the liquid in the tube can be regarded as laminar flow, that is, all liquid at the same distance $\mathrm{r}$ from the center axis of the tube have the same flow velocity $V_{r}$.

Set the viscosity coefficient between the liquid and the tube wall as $\mu$ and we get the following equation:

$$
f=\left.\mu \frac{\partial V_{r}}{\partial r}\right|_{r=R} \cdot 2 \pi R
$$

Remember that the length of the pipe is $\mathrm{L}$ and the pressure difference between the two ends of the pipe is $\triangle \mathrm{p}$. The liquid travels at a constant velocity under these two forces, so by balancing the pressure difference and shear force we get:

$$
\begin{aligned}
& \pi R^{2} \Delta p=\int_{0}^{L} f d x \\
& \Delta p=\frac{-4 \mu L}{\pi R^{4}} Q \quad * \text { we consider } \frac{\Delta p}{Q} \text { as the resistance }\left(\frac{1}{c_{i j}}\right) \text { of the pipeline }
\end{aligned}
$$

Thus, resistance is proportional to $R^{-4}$, and conductance (inverse of resistance) is proportional to $R^{4}$. The energy consumed to build a pipe is proportional to its volume, so, energy $\sim \pi R^{2} L \sim R^{2} \sim C^{\gamma}$ when $\gamma=0.5$, since conductance is proportional to $\mathrm{R}^{4}$.

\section{Kirchhoff's law: $Q_{i j}=C_{i j}\left(p_{i}-p_{j}\right)$}

The establishment of this formula mentioned above is called Kirchhoff's law in a circuit. In order to establish it in the biological network, here is the proof.

We assume that all $C_{i j}$ has been determined at any given moment. Then, we need to find the $Q_{i j}$ to minimize the total 
energy function denoted $f$. The following are our conditions:

$$
Q_{k}=\sum_{i} Q_{i k}=\left\{\begin{array}{cr}
m & k \text { is the source } \\
-1 & k \text { is the sink } \\
0 & \text { else }
\end{array}\right.
$$

For the convenience of expression, the set composed of sources is denoted as $\mathrm{S}_{1}$, the set composed of sinks is referred to as $S_{2}$, and the set composed of neither a source nor a sink is $S_{3}$. By the Lagrange method of seeking conditional extremum, let the function $\mathrm{F}$ be:

$$
\begin{aligned}
F\left(Q_{i j}\right) & =f+\sum_{k \in S_{1}} \lambda_{k} Q_{k}+\sum_{k \in S_{2}} \lambda_{k} Q_{k}+\sum_{k \in S_{3}} \lambda_{k} Q_{k} \\
& =f+\sum_{k \in S_{1}} \lambda_{k} \sum_{i} Q_{k i}+\sum_{k \in S_{2}} \lambda_{k} \sum_{i} Q_{k i}+\sum_{k \in S_{3}} \lambda_{k} \sum_{i} Q_{k i}
\end{aligned}
$$

"where $\mathrm{i}$ and $\mathrm{k}$ are connected nodes

$$
\frac{\partial F}{\partial Q_{i j}}=0
$$

When taking the partial derivative with respect to $Q_{i j}$, expanding sigma notation gives us only two terms that include $Q_{i j}\left(Q_{i j}=-Q_{j i}\right)$ and the rest are all constants. We get: $\frac{2 Q_{i j}}{C_{i j}}+\lambda_{i}-\lambda_{j}=0, \forall(i, j)$

We manipulate the equation algebraically so that $Q_{i j}$ is isolated on one side, and then substitute our conditions for $Q_{i j}$ to get:

* for a given $\mathrm{k}$

$$
\sum_{(k, i)}-\frac{1}{2} C_{k i}\left(\lambda_{k}-\lambda_{i}\right)=\left\{\begin{array}{lr}
m & k \text { is the source } \\
-1 & k \text { is the sink } \\
0 & \text { else }
\end{array}\right.
$$

We can solve the system of linear equations for $\lambda_{i}$; however, we solve the following system for pi for convenience.

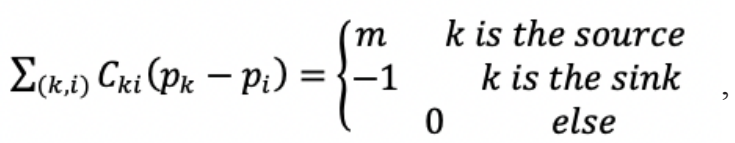

$p_{i}=-\frac{1}{2} \lambda_{i}$

Thus, we have $Q_{i j}=C_{i j}\left(p_{i}-p_{j}\right)$

\section{Principles, ideas, and methods}

The equations mentioned above $\sum_{(k, i)} C_{k i}\left(p_{k}-p_{i}\right)=\left\{\begin{array}{cc}m & k \text { is the source } \\ -1 & k \text { is the sink } \\ 0 & \text { else }\end{array}\right.$ are solvable, where $\mathrm{p}_{\mathrm{i}}$ is an unknown number and $C_{k i}$ is a known coefficient. One of the main conclusions of this section is that although the equation has more than one solution, $\mathrm{p}_{\mathrm{i}}-\mathrm{p}_{\mathrm{j}}$ is unique.

It is not difficult to write the equations in the form of a matrix vector in the form of $\mathrm{A}^{*} \mathrm{P}=\mathrm{I}$, where $\mathrm{A}$ is a $\left(3 \mathrm{~N}^{2}+3 \mathrm{~N}+1\right) \times\left(3 \mathrm{~N}^{2}+3 \mathrm{~N}+1\right)$ matrix and $a_{k k}=\sum_{i} C_{k i}, a_{k l}=-C_{k l}(k \neq l)$

$\mathrm{P}$ is a column vector with dimensions $\left(3 \mathrm{~N}^{2}+3 \mathrm{~N}+1\right) \times 1$, and the element of the $\mathrm{i}$-th row is $\mathrm{p}_{\mathrm{i}}$. 
I is a column vector with dimensions $\left(3 \mathrm{~N}^{2}+3 \mathrm{~N}+1\right) \times 1$, where element $b_{i}$ equals 1 if node $i$ is a source, equals -1 if $i$ is a sink, and equals 0 if $i$ is neither.

The following points can be seen from the element composition of $\mathrm{A}$.

(1) A is a symmetric matrix, because $C_{i j}=C_{j i}$;

(2) The sum of all elements in each row of A is 0 , so A is a singular matrix;

(3) $\mathrm{A}$ is a sparse matrix. In the k-th row, the only non-zero elements in this row are $\mathrm{a}_{\mathrm{ki}}$ ( $\mathrm{k}$ and $\mathrm{i}$ are connected nodes), which are negative values. The diagonal element $a_{k k}$ is the absolute value of the sum of all negative values in the $k$-th row.

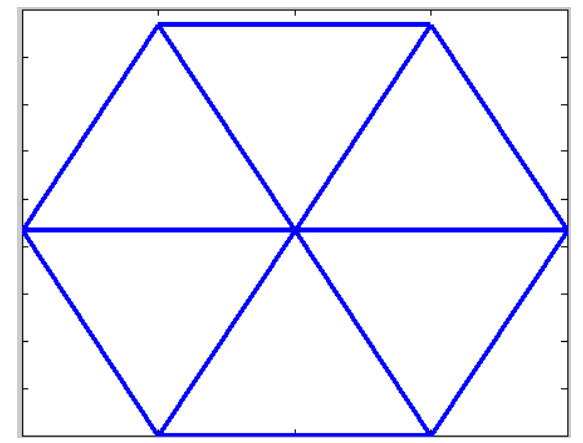

Figure 2. Simplest subset of hexagonal network with seven nodes

We give the simplest network (figure above) and matrix A when each side has only one node in order to grasp the form of the matrix, as shown in the figure below:

$$
\left(\begin{array}{ccccccc}
C_{12}+C_{13}+C_{14} & -C_{12} & -C_{13} & -C_{14} & 0 & 0 & 0 \\
-C_{21} & C_{21}+C_{24}+C_{25} & 0 & -C_{24} & -C_{25} & 0 & 0 \\
-C_{31} & 0 & C_{31}+C_{34}+C_{36} & -C_{34} & 0 & -C_{36} & 0 \\
-C_{41} & -C_{42} & -C_{43} & C_{41}+C_{42}+C_{43}+C_{45}+C_{46}+C_{47} & -C_{45} & -C_{46} & -C_{47} \\
0 & -C_{52} & 0 & -C_{54} & C_{52}+C_{54}+C_{57} & 0 & -C_{57} \\
0 & 0 & -C_{63} & -C_{64} & 0 & C_{63}+C_{64}+C_{67} & -C_{67} \\
0 & 0 & 0 & -C_{74} & -C_{75} & -C_{76} & C_{74}+C_{75}+C_{76}
\end{array}\right)
$$

Because $\mathrm{A}$ is a singular matrix, the original equation either has no solution or the solution has at least 1 degree of freedom.

We can prove that $\mathrm{A}$ is a positive semi-definite matrix by linear algebra.

We know that $r(A)=3 N^{2}+3 N$ (number of rows -1 ). According to the conclusion of linear algebra, $p_{i}-p_{j}$ can be determined by the system of linear equations after determining one variable.

\section{Numerical simulation}

As $Q_{i j}$ has been determined by the system, we can rewrite the energy function.

$$
\begin{aligned}
\text { Minimize } f & =\sum_{(i, j)} \frac{Q_{i j}\left(C_{i j}\right)^{2}}{C_{i j}}+\lambda \sum_{(i, j)} C_{i j}^{\gamma} \\
\frac{\partial F_{\lambda}\left(C_{i j}\right)}{\partial C_{i j}} & =-\frac{Q_{i j}\left(C_{i j}\right)^{2}}{C_{i j}^{2}}+\sum_{(i, j)} \frac{2 Q_{i j}\left(C_{i j}\right)}{C_{i j}} \frac{\partial Q_{i j}}{\partial C_{i j}}+\lambda \gamma C_{i j}^{\gamma-1} \\
& =-\left(p_{i}-p_{j}\right)^{2}+2 \sum_{(i, j)}\left(p_{i}-p_{j}\right) \frac{\partial Q_{i j}}{\partial C_{i j}}+\lambda \gamma C_{i j}^{\gamma-1} \\
& =-\left(p_{i}-p_{j}\right)^{2}+\lambda \gamma C_{i j}^{\gamma-1}+2 \sum_{i} \sum_{j} p_{i} \frac{\partial Q_{i j}}{\partial C_{i j}}-2 \sum_{i} \sum_{j} p_{j} \frac{\partial Q_{i j}}{\partial C_{i j}}
\end{aligned}
$$




$$
\begin{aligned}
& =-\left(p_{i}-p_{j}\right)^{2}+\lambda \gamma C_{i j}{ }^{\gamma-1}+2 \sum_{i} \sum_{j} p_{i} \frac{\partial Q_{i j}}{\partial C_{i j}}-2 \sum_{i} \sum_{j} p_{i} \frac{\partial Q_{j i}}{\partial C_{j i}} \\
& =-\left(p_{i}-p_{j}\right)^{2}+\lambda \gamma C_{i j}{ }^{\gamma-1}+4 \sum_{i} p_{i} \sum_{j} \frac{\partial Q_{i j}}{\partial C_{i j}}
\end{aligned}
$$

For all of the nodes i, $\sum_{j} Q_{i j}$ is a constant, so $\sum_{j} \frac{\partial Q_{i j}}{\partial C_{i j}}=0$. Thus, $\frac{\partial F_{\lambda}\left(C_{i j}\right)}{\partial C_{i j}}=-\left(p_{i}-p_{j}\right)^{2}+\lambda \gamma C_{i j}^{\gamma-1}$. In order to optimize the total energy function with respect to time, we set $\frac{d C_{i j}}{d t}=d_{i j}=\left(-\left(p_{i}-p_{j}\right)^{2}+\lambda \gamma C_{i j}^{\gamma-1}\right)$

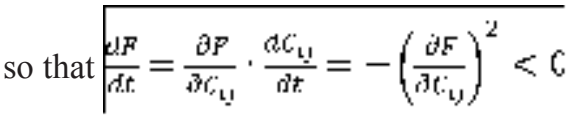

We then use the Runge-Kutta method to minimize $C_{i j}$.

$$
\begin{aligned}
& d_{1}^{(n)}=h\left(C_{i j}^{(n)}\right), \\
& d_{2}^{(n)}=h\left(C_{i j}^{(n)}+0.5 u d_{1}\right) \\
& d_{3}^{(n)}=h\left(C_{i j}^{(n)}+0.5 u d_{2}\right) \\
& d_{4}^{(n)}=h\left(C_{i j}{ }^{(n)}+u d_{3}\right) \\
& C_{i j}^{(n+1)}=C_{i j}{ }^{(n)}+\frac{1}{6} u\left(d_{1}^{(n)}+2 d_{2}{ }^{(n)}+2 d_{3}{ }^{(n)}+d_{4}{ }^{(n)}\right)
\end{aligned}
$$

Thus, we have the terminal network.
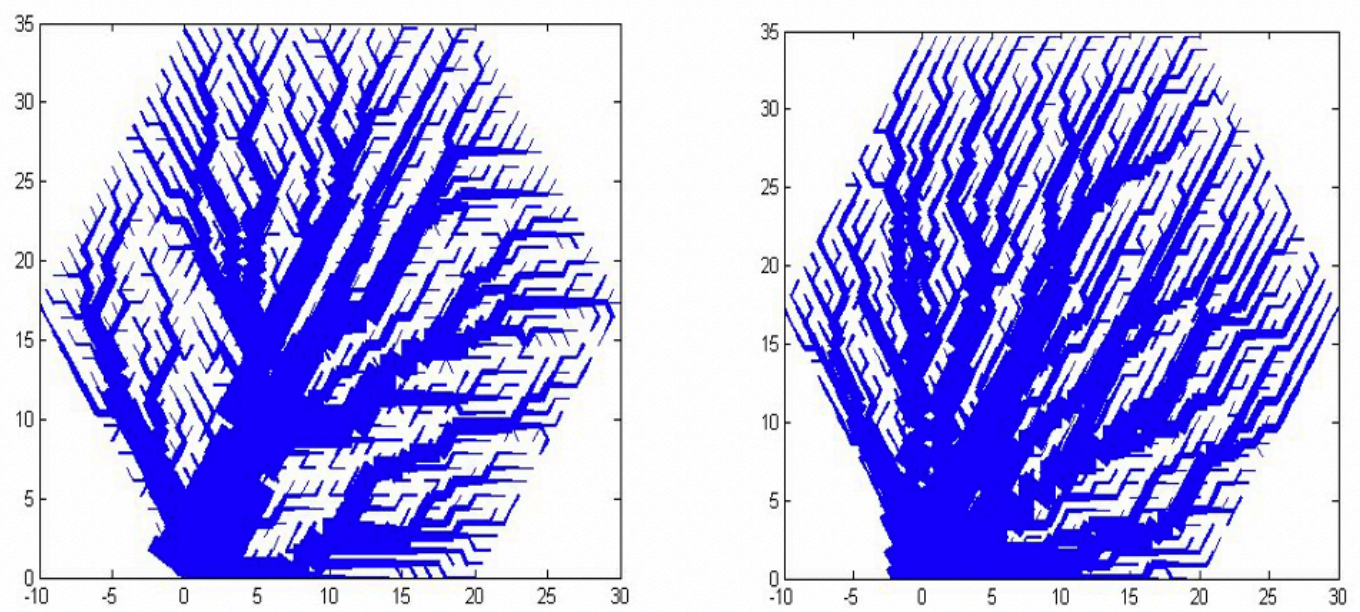

Figure 3. MATLAB simulations of the terminal network over time with step length of 0.01 seconds (left) and 0.1 seconds (right)

The figures above are depictions of the terminal network, where the source node is located at $(0,0)$ in the grid. The right is the same network but given more time to develop and optimize than the left. In this paper, the method we used to search for the minimum energy point is called the negative gradient flow method. Although this method takes longer to reach the terminal network, the network reaches a more minimized total energy consumption compared to other methods. The principle of natural selection tells us that in the process of evolution, organisms always tend to retain mechanisms that are more adapted to their environment. Thus, the advantage of lower energy consumption makes it more likely that slime molds follow the negative gradient flow method instead of other methods when constructing biological networks. 


\section{Conclusion}

There are two main applications of my research:

Organ transplants: For 3D-printed and artificial organ transplants, one major obstacle to its implementation is to imitate vascularization and incorporate the new, artificial organ. Using our model, scientists and doctors can figure out the initial blood vessel radius that minimizes initial energy consumption and toll on the patient's body. Not only does this decrease the need for organ donors and decrease the chance of organ rejections, our model also increases the chance of a successful transplant since the weakened sick body is spending minimal ATP or protein on maintaining the new organ and subsequent blood flow.

Robotics: Large, bipedal robots use liquid pressure to figure out how to walk. On the interior, they require bloodvessel-like systems that carry liquids, where oil is pumped to parts of the robot necessary to walk. For these pipelines, we use our energy consumption model to optimize construction. Mechanical pathways can likewise be optimized and constructed like biological transportation networks.

\section{References}

[1] Eleni Katifori, Gergely J Szollosi, Marcelo O Magnasco. Damage and Fluctuations Induce Loops in Optimal Transport Networks. Physical Review Letters. 2010; 104(4): 048704.

[2] Steffen Bohn, Marcelo O Magnasco. Structure, Scaling, and Phase Transition in the Optimal Transport Network. Physical Review Letters. 2007; 98(8): 088702

[3] Gilbert Strang. Introduction to Linear Algebra (Fifth Edition). Wellesley: Cambridge Press; 2016. 